\title{
STRUCTURAL ELEMENTS WITH TRANSPARENT WOOD IN ARCHITECTURE
}

\author{
D. KATUNSKÝ1,*, J. KANÓCZ ${ }^{2}$, V. KARLA ${ }^{1}$ \\ ${ }^{1}$ Institute of Architectural Engineering, Faculty of Civil Engineering, Technical University of Košice, \\ 04020 Košice, Vysokoškolská 4, Slovakia \\ ${ }^{2}$ Faculty of Arts, Department of Architecture, Technical University of Košice, Košice, Slovakia \\ *E-mail: dusan.katunsky@tuke.sk
}

\begin{abstract}
Transparent wood is a relatively new material. For the first time, it was created in 1992 by a German researcher Siegfried Fink. Back then he turned wood transparent to reveal its specific cavities for analytical purpose. But it was not until between 2015 and 2016 when Professor Lars Berglund from Swedish KTH University and Professor Liangbing Hu from University of Maryland developed a method to remove colour and some chemicals from small wood samples and added polymers to make it transparent. The material then has the higher strength than original wood while having a $90 \%$ transparency. In the future it can be used as an ecological building material which is significantly more biodegradable than plastic or glass. In the present article, examples of the possible use of transparent wood in the construction of structural elements in architecture are given. Examples of beam type load bearing elements and structural parts of the wall structures with use of transparent wood are presented.
\end{abstract}

Keywords: transparent wood, composite material, delignification, methyl methacrylate, epoxy resin

\section{Introduction}

Transparent wood (Fig. 1) is a material which has a great potential to be used in architectural projects worldwide. The composite is not only translucent but also stronger than original wood and polymers that are used at its creation. Its fabrication will be cheaper than fabrication of glass according to scientists who have already researched it and transparent wood will also be more ecologically friendly as they have said.

At a current level of knowledge, it cannot fully replace glass as a transparent material in buildings because right now transparent wood is not really transparent, it is rather translucent, but it can be used in situations where total transparency is not required or where it is actually not wanted, yet a natural light is needed. This composite should also be more resistant than regular wood due to a replacement of lignin by polymer.

On top of that it can also be used as a structural material because of its outstanding properties, and as such, transparent wood could become much desired. Imagining its subtle appearance it would add an incredible lightness to an overall structure of a building. This way new architectures could emerge that would not only be beautiful, functional and durable but also more ecological.

\section{Creation of transparent wood}

So far, transparent wood has been created at only two universities that researched it. It was at Swedish KTH and at Maryland University. Both of those universities had a similar concept of its creation, but used different methods. The concept is to first remove lignin from wood and then to fill it with polymer.

At KTH delignification was achieved using $1 \mathrm{wt} \%$ sodium chlorite $\left(\mathrm{NaClO}_{2}\right)$ with acetate buffer solution (having pH 4.6) at $80^{\circ} \mathrm{C}$. Delignified samples were then cleaned in deionized water. After that, they were dehydrated first with pure ethanol and then with 1:1 mixture of ethanol and acetone. After delignification

This is an open-access article distributed under the terms of the Creative Commons Attribution-NonCommercial 4.0 International License (https://creativecommons.org/licenses/by-nc/4.0/), which permits unrestricted use, distribution, and reproduction in any medium for non-commercial purposes, provided the original author and source are credited, a link to the CC License is provided, and changes - if any - are indicated. 


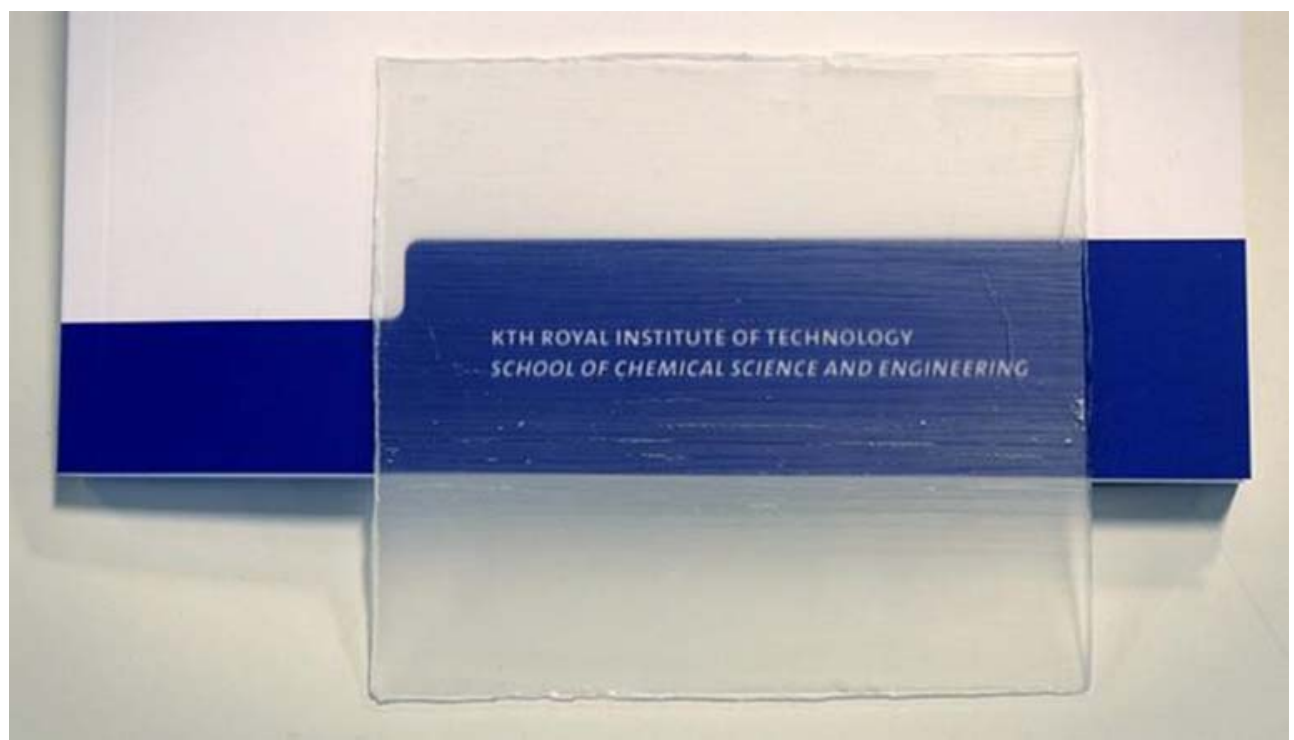

Fig. 1. Transparent wood sample created at KTH [1]

was over, prepolymerized solution of methyl methacrylate (MMA) was inserted into wood in vacuum [2].

At Maryland, delignification was a two-step process. The first step was inserting wood samples into a boiling solution of $\mathrm{NaOH}$ and $\mathrm{Na}_{2} \mathrm{SO}_{3}$. It was then followed by inserting partly dignified samples into a solution of $\mathrm{H}_{2} \mathrm{O}_{2}$ to remove the remaining lignin. After that, epoxy resin was infiltrated into delignified wood using repeated vacuum and de-vacuums [3].

\section{Properties of transparent wood}

As of yet, only some thin samples of transparent wood have been fabricated. However, their properties are very well examined. Researches have mainly studied their optical and mechanical properties. From those studies we know about the following findings.

Transparent wood of various thicknesses shows different optical properties. The thinner the sample, the higher its optical transmittance. It is because transparency is dependent on a volume fraction of cellulose. At KTH the highest optical transmittance has been achieved with $0.7 \mathrm{~mm}$ thick sample and it has been a value of $90 \%$. The lowest has been approximately $40 \%$ with $3.7 \mathrm{~mm}$ thick sample [2]. In Mary- land, they have examined how properties of transparent wood depend on a cut of original wood, meaning they have been studying properties of radially and longitudinally cut wood. As a radially cut wood has a lower cellulose volume fraction, they have achieved $90 \%$ optical transmittance with $2 \mathrm{~mm}$ thick sample and around $80 \%$ with the same thickness of longitudinally cut wood. Another optical property they both have researched is optical haze of the samples [3]. At KTH they have measured $60 \%$ to $80 \%$ values of haze, while it has been higher in thicker samples [2]. In Maryland they have measured haze of $90 \%$ for longitudinally cut wood and surprisingly close to $100 \%$ for radially cut wood. Radially cut wood does have a lower cellulose volume fraction, but an important factor here is an arrangement of wood cells that has created the higher haze [3].

Transparent wood has also shown outstanding mechanical properties. At KTH, they have figured out that they are better for transparent wood, than they are for both, natural wood and PMMA. The elastic modulus for transparent wood with a cellulose volume fraction of $5 \%$ was $2.05 \pm 0.13 \mathrm{GPa}$ (it was 1.80 $\pm 0.18 \mathrm{GPa}$ for PMMA and $0.22 \pm 0.08 \mathrm{GPa}$ for delignified wood). Tensile testing in longitudinal direc-

Table 1. Comparison of mechanical properties of natural radially cut wood, natural longitudinally cut wood, transparent radially cut wood and transparent longitudinally cut wood [3]

\begin{tabular}{lccc}
\hline & $\begin{array}{c}\text { Strength } \\
(\mathrm{MPa})\end{array}$ & $\begin{array}{c}\text { Modulus } \\
(\mathrm{GPa})\end{array}$ & $\begin{array}{c}\text { Toughness } \\
\left(\mathrm{MJm}^{-3}\right)\end{array}$ \\
\hline Natural radially cut wood & 4.46 & 0.19 & 0.10 \\
Transparent radially cut wood & 23.38 & 1.22 & 0.59 \\
Natural longitudinally cut wood & 42.72 & 5.78 & 0.33 \\
Transparent longitudinally cut wood & 45.38 & 2.37 & 1.20 \\
\hline
\end{tabular}


tion of transparent wood with cellulose volume fraction of $19 \%$ measured its tensile strength as $90.1 \pm 10$ MPa with a modulus of $3.59 \pm 0.27 \mathrm{GPa}$ (for PMMA it was $44.1 \pm 9.5 \mathrm{MPa}$ ) [2]. At Maryland they have also examined mechanical properties of the samples and found that both radially cut and longitudinally cut wood are both stronger than their natural counterparts and that transparent longitudinally cut wood is about two times as strong as transparent radially cut wood (Table 1) [3].

\section{Proposed architectural elements using transparent wood}

The main focus of this article is to present some fresh ideas of how transparent wood can be used in architecture and building industry or civil engineering. When talking about transparent wood, the first thing which comes to mind is to use it as a façade material, but it is at least as much interesting to use the composite structurally. On the following pages we will examine both cases and provide the reader with some proposed examples of how we imagine the shapes of transparent wood in architecture might look like and what we actually plan to research further in the future.

\subsection{Transparent wood façades}

Transparent wood is to be used on façades in such cases when there is a requirement for sunlight to illuminate the interior, but privacy is just as much required. It is because of the high values of optical haze in transparent wood, what makes it let the light in, but clouds the view, thus can be used for those cases.

There are still many questions about transparent wood that need to be answered. In the case of transparent wood façades it is mainly a question of durability and whether we need to protect the material and if we do, then how? This is something that will have to be researched, because right now, we do not have any answers. As of now, we presume that the composite will be more durable than natural wood, because the lignin, which is removed in transparent wood and replaced by polymer, usually causes the most severe problems considering degradation of wood.
Another big question is whether we can also turn thick samples of wood transparent? The recent updates from KTH show that it might be possible. They have improved the process of transparent wood fabrication and delignification is no more needed, instead they have found a way to modify lignin and have made the whole process much more ecologically friendly while reducing the time needed for transparent wood to be created [4]. Still, the thicker the sample is, the less transparent it becomes. Therefore, there are actually two ways, to make a transparent wood façade. The first one is to produce wall units of transparent wood, which will be thick enough to act structurally. The disadvantage it poses is less light coming into an interior. An upgrade here could be to make such façades as origami structures, what could reduce the thickness of walls thanks to geometry of such structures. The second option is to use much thinner samples and make transparent wood façade analogically to structural glass façade, while it would be quite interesting to use thick samples of transparent wood as columns of the façade, so architectonically, it would be very novel creation that would provide us with interesting light conditions as well as with a very much desired aww effect. A modification of the second option is to create frames, similar to window frames, out of thick transparent wood and insert a thin transparent wood plate into this frame. Compared to glass, transparent wood has better insulating properties, which is yet another benefit of using it instead.

Interesting aspect of transparent wood is also its colour. The new method of fabricating transparent wood researched at $\mathrm{KTH}$, which is leaving the lignin in the wood, does not remove all the colour of wood. Therefore different species of wood have slightly different colours. They have created transparent wood from balsa, pine, birch and ash wood. Balsa is almost white, while other species have a yellowish tone. Ash is just slightly yellow, birch is even a little more and pine has quite saturated yellow tone [4]. Being it like that, it can create some very interesting patterns on façades, just by using different species of wood. Another benefit is that interior space will be flooded by a very positive yellow light, which might improve the mood of people inhabiting it.

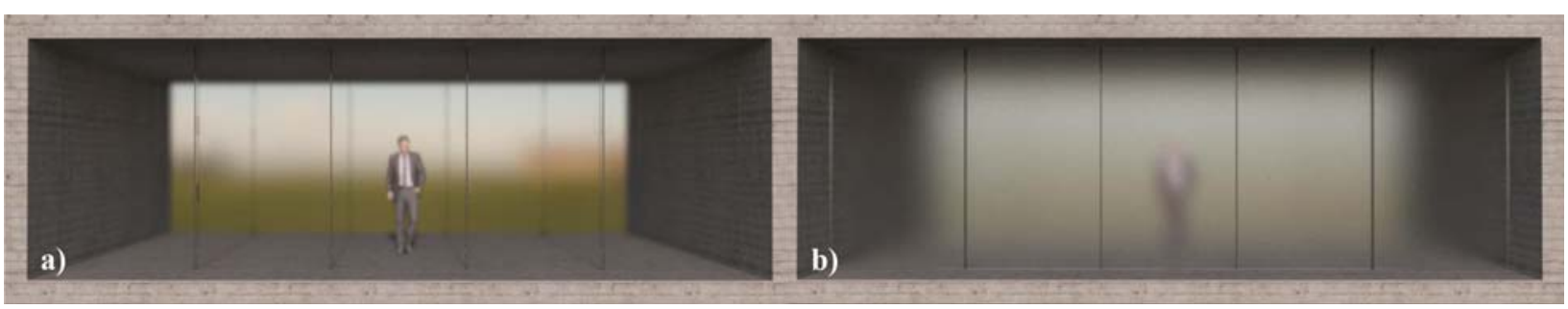

Fig. 2. Thin structural (a) and thick wall (b) transparent wood façades rendering [5] 


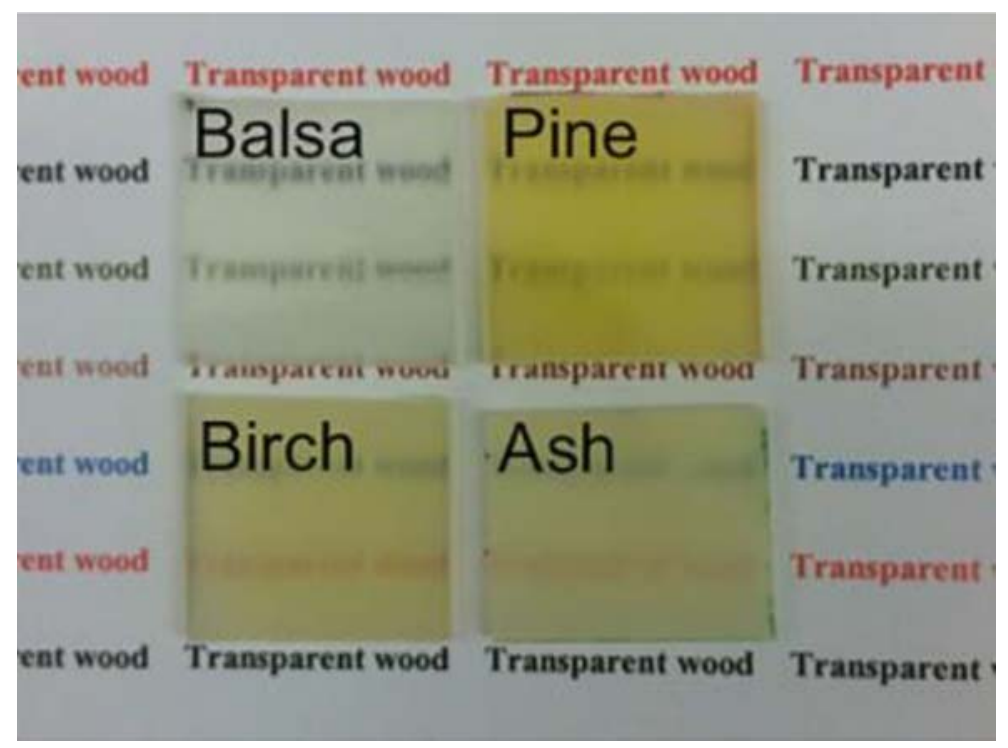

Fig. 3. Various species of transparent wood [4]

\subsection{Structural elements using transparent wood}

It is a very interesting fact about transparent wood that it is actually a stronger material than a natural wood it was made of and also stronger than polymer, which was used at its creation. For this reason, structural elements made of transparent wood are not only an el-

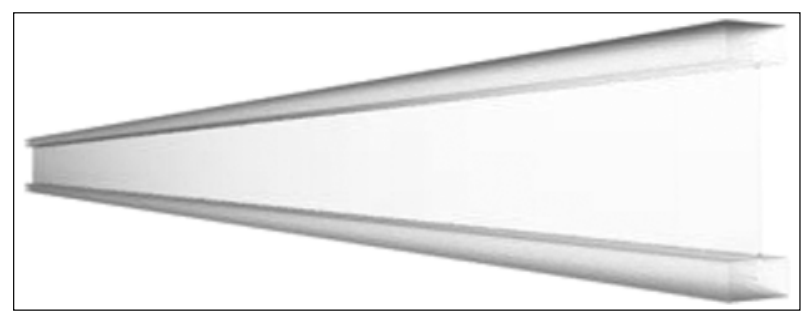

Fig. 4. Transparent wood I beam - rendering

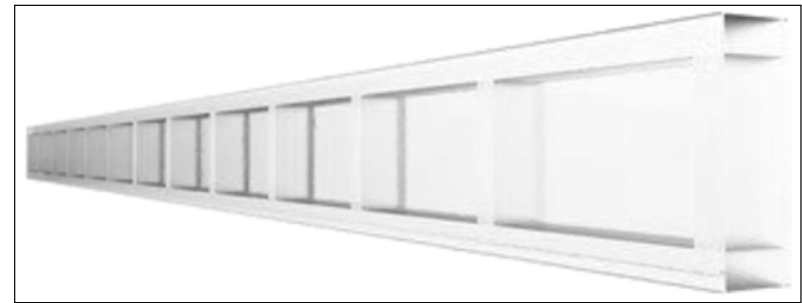

Fig. 5. Transparent wood Vierendeel box beam - rendering

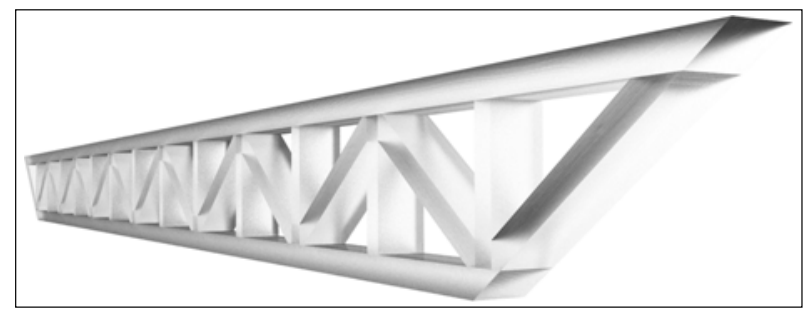

Fig. 6. Transparent wood truss - rendering egant, but a very smart solution. If there is a chance now to fabricate large pieces of transparent wood, we can just use big sections of transparent wood and make beams out of them. The only problem is that such beams would not transmit as much light. This is the reason, why we propose different beams, using thin sections of transparent wood, so the appearance of the whole structure will be much lighter and more attractive.

Firstly, we would like to research "I" beams, where the web of those beams is to be made of thin transparent wood and flanges are to be made either of a laminated veneer lumber or thick transparent wood members. This is, as we believe, the lightest possible version of transparent wood beams in the means of appearance. Architectonically, a structure made using such beams would almost seem to be floating or levitating in space, which might become much desired.

Another option is to create box beams. They would be created in a similar fashion as "I" beams. Flanges could again be made of laminated veneer lumber or thick transparent wood members and webs would be made of thin transparent wood panels. These beams are not as light (although still pretty light), but can support bigger weights. The variation of the box beams is to create a Vierendeel truss (again, either by using natural or thick transparent wood) and add thin transparent wood panels at its both sides. Out of all the mentioned it is a least light variant, but the firmest.

Transparent wood trusses are yet another way to use transparent wood structurally. Of course, the thicker members will have to be used to create them, but not as thick, as it would require a single massive beam. So, compared to that, it is still a lighter structure. Such 
trusses can be all transparent wood, or only a flange would be made of transparent wood and it would then work together with steel cable to achieve a much lighter structure overall.

Of course, using massive transparent wood beams is also an option. For this case, we believe it would have been for the best to get inspired by composite wood materials, which perform better than solid wood. This way transparent glued laminated timber could emerge in the building industry, as well as transparent cross laminated timber, transparent laminated veneer lumber, transparent plywood and maybe even transparent oriented strand board.

All of the proposed solutions, being it using transparent wood for façades or as a structural material will undoubtedly require an industrialisation of the fabrication of transparent wood, but that way it would become much easier to actually use it even in large scale projects and to popularize its use worldwide.

\section{Visions for the future}

Once the fabrication of transparent wood will become industrialised, many products using this material will emerge. Probably many more than those we proposed in this article. We believe that one day, it will be possible to build an entire building only using elements created with transparent wood. The best way to explain our visions is to describe a design, which already incorporated transparent wood.

In 2016, we were attending an architectural competition [5]. Its aim was to design a beacon on Sapphire Coast in Australia. It was encouraged by the competition rules to create an innovative and sustainable design. Our concept was to design a building, which would resemble a sail and to use wood as the main structural material. During the design process, we felt that to make the design the most believable, we needed as much transparency as we could get, so the feeling of a sail resemblance would be undisturbed. Just at

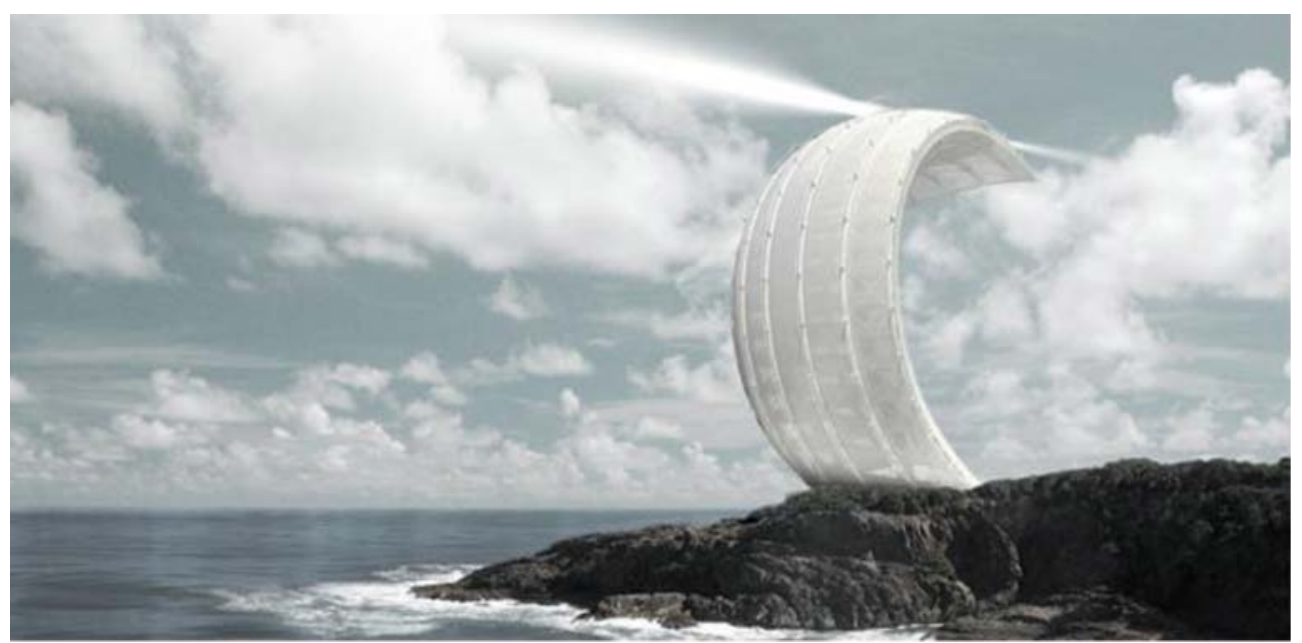

Fig. 7. Tathra Beacon exterior rendering [5]

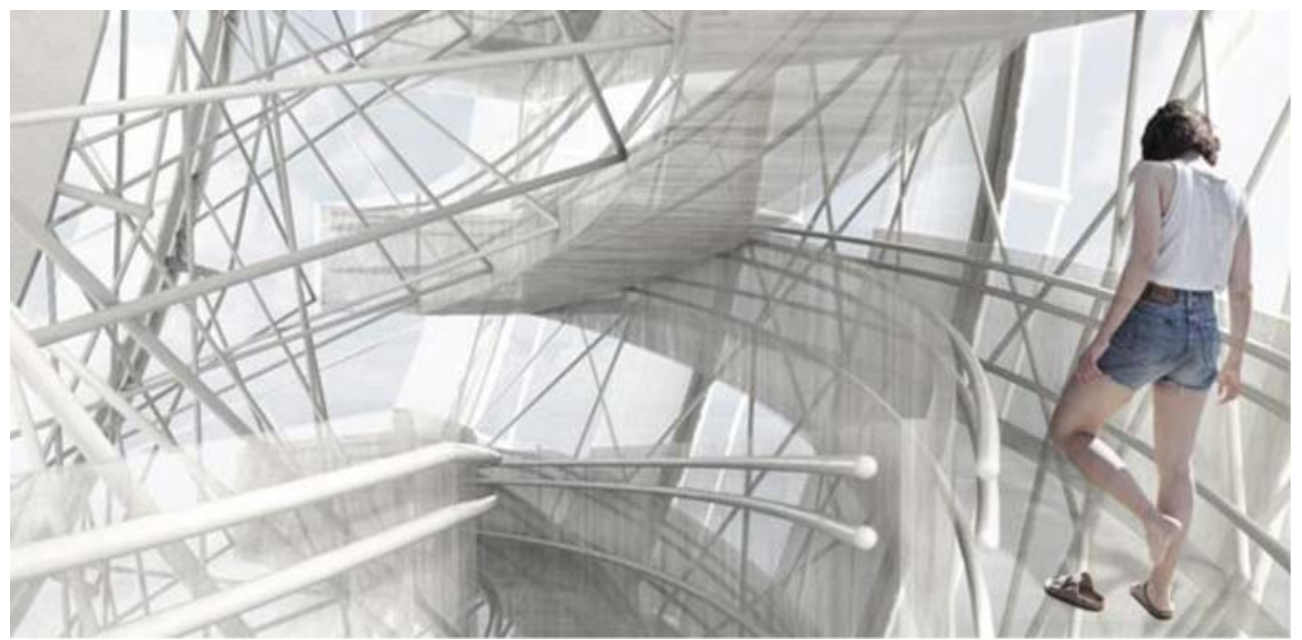

Fig. 8. Tathra Beacon interior rendering [5] 
that time, there was an article published by KTH about an invention of transparent wood. It quickly became our main structural material for the design.

We proposed to use transparent wood for the main loadbearing beams as well as ramps. The main loadbearing beams were supposed to be transparent glued laminated timber reinforced by a steel cable on the side of a beam in tension. On those beams, there would be ramps fixed to them using steel rods. Railings of these ramps would also act as girders. They would be made using transparent wood. It would either be transparent glued laminated wood or transparent cross laminated timber. Floor of the ramps was to be made of transparent cross laminated timber, as well. Transparent wood would also be used for certain furniture elements.

Aside from those constructions, we wanted to use ETFE foils for façade, concrete in foundations and roof and curved glass panels were supposed to act as a Fresnel lens for the beacon light. That is, what this project required, but transparent wood could be used even in a much wider applications in different buildings. Mainly transparent wood façades could definitely be an interesting structure, which could take many shapes, they could also be colourfully playful and one could also play with the levels of transparency at different parts of the façade.

The thing that will require much attention considering transparent wood in the future, will definitely be a detailing of fixtures. As the material is transparent, fixtures will have to be thought of very precisely, because they will be visible even inside the material and as such, they will also have to be executed very precisely, both in a factory and on site.

\section{Conclusion}

Transparent wood is a very new material and is still being researched, but has a great potential to become an important building material. Its translucency makes it an interesting choice to be used in façades and its mechanical properties are outstanding and predeter- mine it to be used as a structural material. In this article, we proposed a few possible applications of usage of transparent wood in both those cases. We tried to show that even if it is not yet a material that is fully developed, it has a huge potential and should be researched further. After the recent updates from KTH, it is obvious that better ways of fabricating transparent wood are to come and we believe that in time its production will become industrialised. Meanwhile we feel that we need to focus on research of transparent wood applications in building industry and plan to research the ideas presented in this article.

\section{Acknowledgements}

The contribution resulted from the solution of research projects VEGA /0674/18 and VEGA 1/0538/16 supported by the Scientific Grant Agency of the Ministry of Education, Science, Research and Sport of the Slovak Republic and the Slovak Academy of Sciences.

\section{References}

[1] http://www.constructionmanagermagazine.com/news/ wood-based-gla1ss-altern2ative-ideal-solar-pan3els/

[2] Yuanyuan Li, Qiliang Fu, Shun Yu, Min Yan, Lars Berglund (2016), Optically transparent wood from a nanoporous cellulosic template: Combining functional and structural performance. Biomacromolecules, 17, 1358-1364.

[3] Mingwei Zhu, Jianwei Song, Tian Li, Amy Gong, Yanbin Wang, Jiaqi Dai, Yonggang Yao, Wei Luo, Doug Henderson, Liangbing Hu (2016), Highly anisotropic, highly transparent wood composites. Advanced Materials, 28(26), 5181-5187.

[4] Yuanyuan Li, Qiliang Fu, Rojas R., Yan M., Lawoko M., Berglund L (2017), A new perspective on transparent wood: Lignin-retaining transparent wood. ChemSusChem. 10. 10.1002/cssc.201701089.

[5] Karl'a V., Kanócz J., Mertová M. (2016), View tower in Tathra. The 2016 MNPG Arch Competition. http://mnpgarch.com/archives/427. 